\title{
Multistatic human micro-Doppler classification with degraded/jammed radar data
}

\author{
Francesco Fioranelli, Jarez Patel \\ School of Engineering \\ University of Glasgow \\ Glasgow, UK \\ francesco.fioranelli@glasgow.ac.uk
}

\author{
Sevgi Zubeyde Gürbüz \\ Department of Electrical and Computer \\ Engineering \\ University of Alabama Tuscaloosa \\ Tuscaloosa, AL, United States
}

\author{
Matthew Ritchie, Hugh Griffiths \\ Department of Electronic and \\ Electrical Engineering \\ University College London \\ London, UK
}

\begin{abstract}
This paper investigates the classification performance of using multistatic human micro-Doppler radar data that have been degraded by some form of jamming. Two simple cases of Signal-to-Noise Ratio (SNR) degradation and nulling of a sub-set of the available radar pulses are considered for these initial results, leaving more complex forms of jamming for future work. Experimental data collected with a multistatic radar are used in this study, aiming to classify 7 similar human activities, when individual subjects are walking carrying different objects. The results show that the use of multistatic radar data can provide resilience to the effect of the data degradation, thanks to the redundancy and additional information available from multiple radar nodes.
\end{abstract}

Keywords-radar micro-Doppler, target classification, machine learning, radar data degradation and jamming

\section{INTRODUCTION}

Human micro-Doppler information has been used for classification of targets of interest in many applications. These include classification of activities of daily living, fall detection, and gait analysis [1-3]; classification of people vs animals for ground surveillance radar [4]; personnel identification [5-6]; and potential armed vs unarmed classification [7-8]. The specific information contained in the micro-Doppler signatures is related to the pattern of small movements of limbs and torso, which can be used as a proxy to identify specific actions and even specific people.

One of the limitations of classification approaches based on radar micro-Doppler is its dependence on the cosine of the aspect angle between the radar line-of-sight and the targets' trajectories. When this angle is high, i.e. when the targets are moving along tangential trajectories, the microDoppler signature can be severely attenuated degrading the classification performance [9-10]. Multistatic radar can address this problem, by providing information from multiple, spatially diverse radar nodes, so that at least one of them can observe the targets from a favourable aspect angle $[7,11]$.

The majority of research works on micro-Doppler based classification, at least those in the open literature, assume that the radar signatures are of good quality. There may lower or higher Signal-to-Noise Ratio (SNR) depending on the conditions on which the data were acquired, but, to the best of our knowledge, only few works explore the effect of interruptions and/or degradation that may affect the radar data with different patterns. One piece of work is [12], where the authors considered the effect of interruptions in the available monostatic radar data and possible interpolation techniques to counteract that effect.
In this work, we consider the effect of simple degradation patterns on the available multistatic radar data and compare the classification performance for identifying 7 different human activities between monostatic and multistatic data, with different levels of degradation. The radar data are degraded by altering the SNR of a sub-set of the available radar pulses, or by substituting some of them with zeros. The former case may be associate to the unintentional jamming from a competing transmitter, radar or communication, operating in the same spectral band and the same time; the latter case may be associated to malfunctioning or missed transmission/synchronisation between multistatic radar nodes that make some pulses unusable.

We show preliminary results that demonstrate how, perhaps unsurprisingly, information available from different multistatic radar nodes can support good classification performances, even in case of severe degradation of SNR to many pulses and for a relatively challenging classification problem with 7 similar activities. More complicated and realistic types of jamming, for example those involving retransmission of modified and mismatched versions of the real radar pulses will be considered as an expansion to this work [13]. Furthermore, techniques that can identify the degradation and/or the counterfeiting of available radar data and then restore the original, authentic information will be considered for future work. These may include the use of dictionary learning and/or transfer learning, which can regenerate some of the missing or altered information in the data for classification based on previous knowledge of the training data, at least to the point that classification performances are restored to acceptable levels.

The remainder of this paper is organised as follows. Section II describes the radar used for data collection and the experimental setup. Section III describes the data processing and discusses some initial results. Finally, section IV draws conclusions and outlines possible future work.

\section{EXPERIMENTAL SETUP AND DATA COLLECTION}

The data analysed in this paper were collected in March 2016 at the UCL sports grounds to the North of London, in an empty field relatively free of clutter and obstacles. The radar system was the multistatic, coherent pulse radar NetRAD. This consists of three separate but identical nodes, operating at S-band with $45 \mathrm{MHz}$ signal bandwidth. The signal is a linearly modulated up-chirp waveform with duration equal to $0.6 \mu \mathrm{s}$ and $5 \mathrm{kHz}$ Pulse Repetition Frequency (PRF). The radar transmits approximately 200 $\mathrm{mW}$ and the antennas used at the transmitter and receiver had approximately $18 \mathrm{dBi}$ gain and beam-width of $18^{\circ}$ in both the horizontal and vertical plane [14]. 
The three radar nodes were deployed as shown in Fig. 1. One node was used as monostatic transceiver with transmitted and received $\mathrm{V}$ polarisation, and two nodes as bistatic receivers; one of them was co-located with the transceiver but set to record $\mathrm{H}$ polarisation (cross-polarised data), and the other was located with a baseline of $50 \mathrm{~m}$ from the transceiver and set to record $\mathrm{V}$ polarisation (co-polarised data). The targets were 4 different subjects who would walk towards the baseline of the radar, starting from a distance of approximately $90 \mathrm{~m}$. The key body parameters of these subjects were $1.87 \mathrm{~m}$, male, average body type for person A, $1.60 \mathrm{~m}$, female, average body type for person $\mathrm{B}, 1.78 \mathrm{~m}$, male, slim body type for person $\mathrm{C}$, and $1.70 \mathrm{~m}$, male, average body type for person $\mathrm{D}$. Seven different walking activities were considered by carrying different objects with different postures, namely:

1. Normal walking with nothing carried

2. Walking with a rucksack strapped on one shoulder

3. Walking with a rucksack strapped on both shoulders (shown in Fig. 1, right)

4. Walking with a metallic pole slung on one shoulder

5. Walking with a metallic pole held with both hands to the front

6. Walking with a metallic pole slung on one shoulder and rucksack strapped on both shoulders (shown in Fig. 1, middle)

7. Walking with a metallic pole held with both hands to the front and rucksack strapped on both shoulders

These activities were chosen to be similar one to another and investigate the possibility of using radar micro-Doppler signatures to classify them. The expectation was that the presence of the rucksack and/or the metallic pole (mimicking a rifle) would alter the swinging of torso and limbs of the walking subjects, with respect to the case of free, empty-
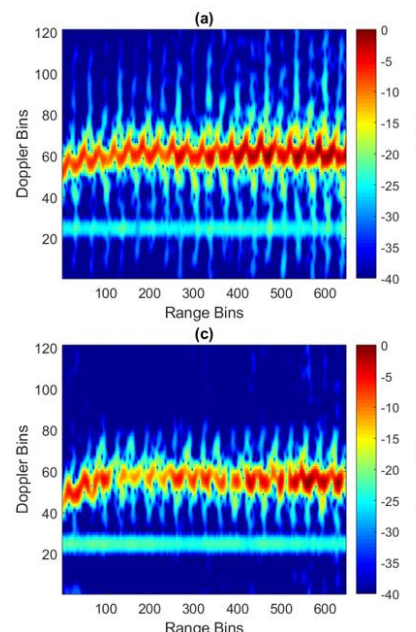

handed walking. A total number of 840 data recordings was collected, with 4 subjects, 7 activities, 3 radar nodes, and 10 repetition for each activity. Each individual data recording had a duration of $10 \mathrm{~s}$.

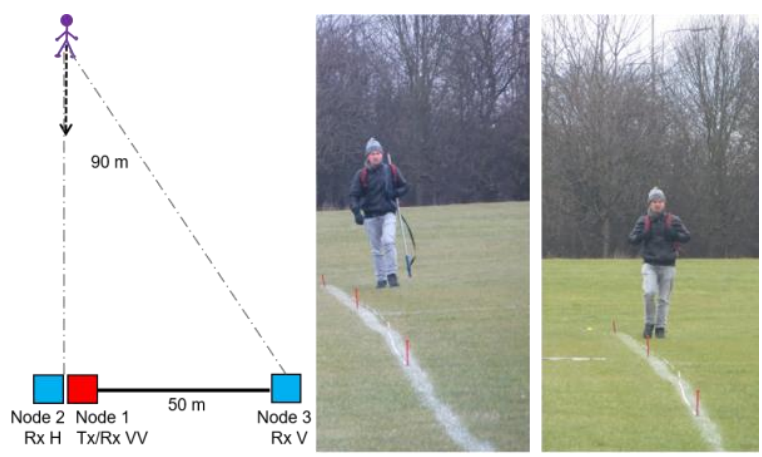

Fig. 1. Sketch of experimental setup and example pictures of subjects performing the activities

\section{DATA PROCESSING AND DATA ANALYSIS RESULTS}

Micro-Doppler signatures were extracted from each recording using Short Time Fourier Transform (STFT) to generate spectrograms. A $0.3 \mathrm{~s}$ Hamming window was used, with $95 \%$ overlap. The resulting spectrograms were 10s long and divided into segments of $1.5 \mathrm{~s}$ duration prior to feature extraction, in order to increase the number of feature samples available for the classifiers. Figure 2 shows examples of the whole spectrograms (10s) for 4 different activities performed by the same subject. One can see the differences in terms of spread of the signature around its centre of mass, due to the different amount of movements of limbs in the different activities. The light blue line around Doppler bin 20 is the residual static clutter near $0 \mathrm{~Hz}$.
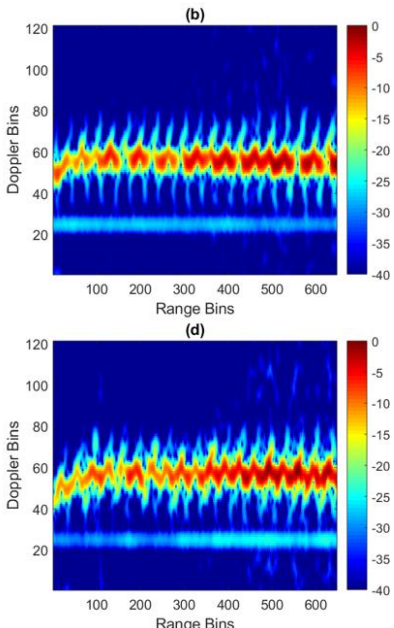

Fig. 2. Examples of spectrograms for 4 activities: (a) walking, (b) walking while carrying a rucksack on the back with both straps on, (c) walking while carrying a metal post with both hands, and (d) walking while carrying both rucksack and metal post
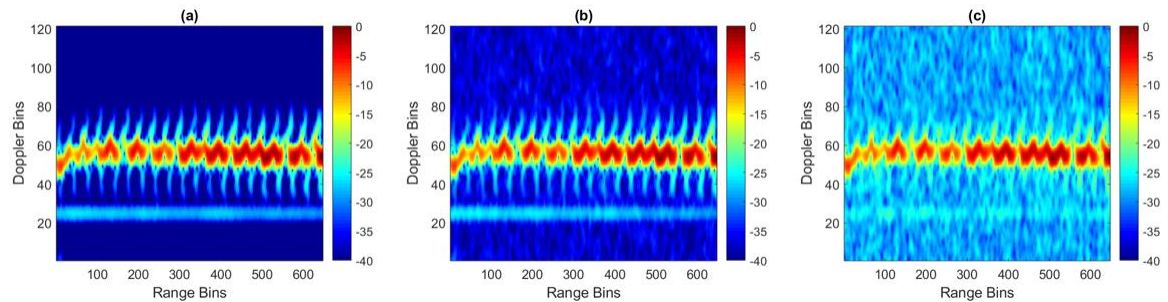

Fig. 3. Examples of spectrograms where $100 \%$ of the pulses were altered to change their SNR: (a) $20 \mathrm{~dB}$, (b) $10 \mathrm{~dB}$, and (c) $1 \mathrm{~dB}$ 


\section{A. Data degradation}

Two different approaches were implemented to simulate some form of degradation on the available radar data. In the first approach, a randomly selected set of pulses were modified in their Signal-to-Noise-Ratio (SNR) by using a suitable MATLAB tool which estimates the current SNR of the pulse and adds or removes noise to reach a desired level of SNR. We have performed different tests with a different percentage of radar pulses affected, namely $10 \%, 20 \%, 40 \%$, $60 \%, 80 \%$, and $100 \%$, and different levels of SNR, namely $20 \mathrm{~dB}, 15 \mathrm{~dB}, 10 \mathrm{~dB}, 5 \mathrm{~dB}$, and $1 \mathrm{~dB}$. In the second approach, a randomly selected set of pulses were modified by changing their values to zeroes. Different tests were performed by nulling a different percentage of pulses, namely $10 \%, 20 \%$, $40 \%, 60 \%, 80 \%$, and $100 \%$.

The degradation was implemented in the range-time domain, prior to performing STFT and spectrogram generation. Figure 3 shows examples of the same dataset where $100 \%$ of the available pulses were modified in their SNR as per approach one described above. In the severe degradation case of $1 \mathrm{~dB}$ of SNR, the contribution from the limbs in the spectrogram (light blue colour) is rather difficult to be distinguished in the figure, at least empirically by eye.

In both approaches, the degraded pulses were selected randomly for each dataset, meaning that simultaneous multistatic data at different radar nodes will have different patterns of affected pulses. In some cases the same radar pulse may be affected by degradation for multiple nodes, in others a degraded pulse recorded at one node is not degraded at others. These are very simple examples of degradation, to model for example those due to possible unintentional "jamming effects" from competing communication systems in the same frequency bands (e.g. Wi-Fi reducing the SNR of some radar pulses), or due to possible malfunctioning of the radar (e.g. some pulses be considered as zero, non-received). More complex forms of jamming, for example those implying re-transmission or replicas of the actual radar pulses or mismatching of the matched filter, will be considered in future work [13].

\section{B. Feature extraction and classification}

Simple features were extracted from each segment of spectrogram based on the centroid and the bandwidth of the micro-Doppler signature. The centroid represents the dynamics of centre of mass of the signature over time, whereas the bandwidth tracks the spread of the signature around the centroid over time [8]. Simple statistical moments of these two parameters were considered as features, namely the mean, the standard deviation, the kurtosis, and the skewness, generating 8 features in total for this 7-class identification problem.

Four simple classifiers were considered in this work, namely Naïve Bayes (NB), nearest neighbour with 3 neighbours (KNN), and Support Vector Machine (SVM) with radial basis functions in the kernel. The available data were randomly partitioned in a $70 \%$ set for training and $30 \%$ for testing, with 10 repetitions of the process for crossvalidation. The average accuracy across the 10 repetitions was recorded as performance metric.

A simple wrapper method was considered for feature selection, by evaluating the performance of all possible combinations of the available 8 features and noting the best combination [10]. In this case, the same feature selection scheme was applied at all multistatic radar nodes, i.e. the same feature combinations is used for data recorded at each node, without investigating benefits of "feature diversity" at this preliminary stage [7]. The optimal set of features, that is the one providing the highest accuracy for the 7-class classification problem, is evaluated for every combination of parameters related to the degradation, i.e. for every combination of level of SNR and number of radar pulses affected.

Multistatic data were used jointly by applying a decisionlevel fusion scheme. Three independent classifiers were implemented for each radar node generating partial decisions with related levels of confidence. These were then fused together by either selecting the class label indicated by at least 2 of the 3 radar nodes, or selecting the class label with the highest confidence among the 3 nodes if there was no majority decision available. Alternative fusion methods at decision level, feature level, and signal level are possible, but these are not considered for the initial results in this work.

\section{Data analysis and results}

In this section, we report some initial classification results for different levels of degradation of the original radar data. Figure 4 shows two heat maps tables, where the average classification accuracy is reported comparing the case of monostatic vs multistatic information for SVM classifier. The same colour scale is applied to both tables, and the results for different values of SNR and percentages of radar pulses affected by the degradation. As expected, the worst accuracy (approximately 62-64\%) is achieved when the SNR is low, at $1 \mathrm{~dB}$, in the monostatic case, even for a relatively small percentage of pulses affected. In general, there is an improvement in accuracy of approximately 10$14 \%$ when using multistatic information in comparison with using only monostatic data. It is interesting to observe that for low values of SNR, 1 and $5 \mathrm{~dB}$, the accuracy decreases when more radar pulses are degraded, whereas the opposite effect is noted for a high value of SNR, at $20 \mathrm{~dB}$ for example. One possible explanation of this effect is that imposing high values of SNR may have actually improved the original radar recordings rather than degrading them, as the initial SNR before any processing was lower. This effect is still being investigated in current work, also considering that these variations in accuracy are small, around 1-2\%, hence some questions on their significance are still outstanding.

In Figure 5 we show an example of comparison of accuracy for 4 different classification approaches, namely all the 3 classifiers (NB, KNN, SVM) using multistatic data and the SVM with only monostatic data, as a function of SNR. In all cases, $80 \%$ of the radar pulses were affected by the SNR modification. As expected, SVM with multistatic data achieves the highest accuracy for all SNR values. It is interesting to note that a relatively simple classifier (KNN with 3 neighbours) can match in performance the more complicated SVM when the former uses multistatic data and the latter only monostatic data. Even in the best case the highest accuracy is in the range of $85 \%$, significantly higher than random decision for the 7-class problem (about 14.3\%) but still improvable. We envisage that the application of the most recent classification approaches based on deep learning and data-driven features [2, 15], combined with the availability of multistatic data, can further improve these 
results and provide added resilience to the classification performances in case of data degradation.

In Figure 6 we report a heat map table with the accuracy obtained with the 4 classification approaches when different percentages of radar pulses are replaced with zeroes. The effect of this appears to be rather limited, with variations in average accuracy of about $1 \%$ between few (10\%) and many $(80 \%)$ pulses replaced by zeros. Much more significant, as already observed for figure 5 , is the choice of the classifier and the availability of multistatic data. Additional work to understand and investigate these results is undergoing, as more severe degradation in performances were expected when a large number of pulses are replaced by zeros. A possible explanation for the resilience in performance may be that, as the pattern of degraded pulses is random (so not necessarily a long sequence of nulled pulses, but random "puncturing" of the data) and the PRF of the data is very high, the required information for generating acceptable micro-Doppler signatures is still there.

Finally, Figure 7 shows an example of confusion matrix to see where the most likely misclassification events appear. The case selected is one of those with the highest accuracy, on average $85.4 \%$, with $100 \%$ of the pulses altered to have $20 \mathrm{~dB}$ SNR. There is no clear pattern of misclassification between the 7 different activities, and the errors appear to be evenly distributed outside the diagonal of the matrix. This can pose a challenge to further optimise the classification performance, even if it should be noted that the classification approaches used in this paper are rather easy.
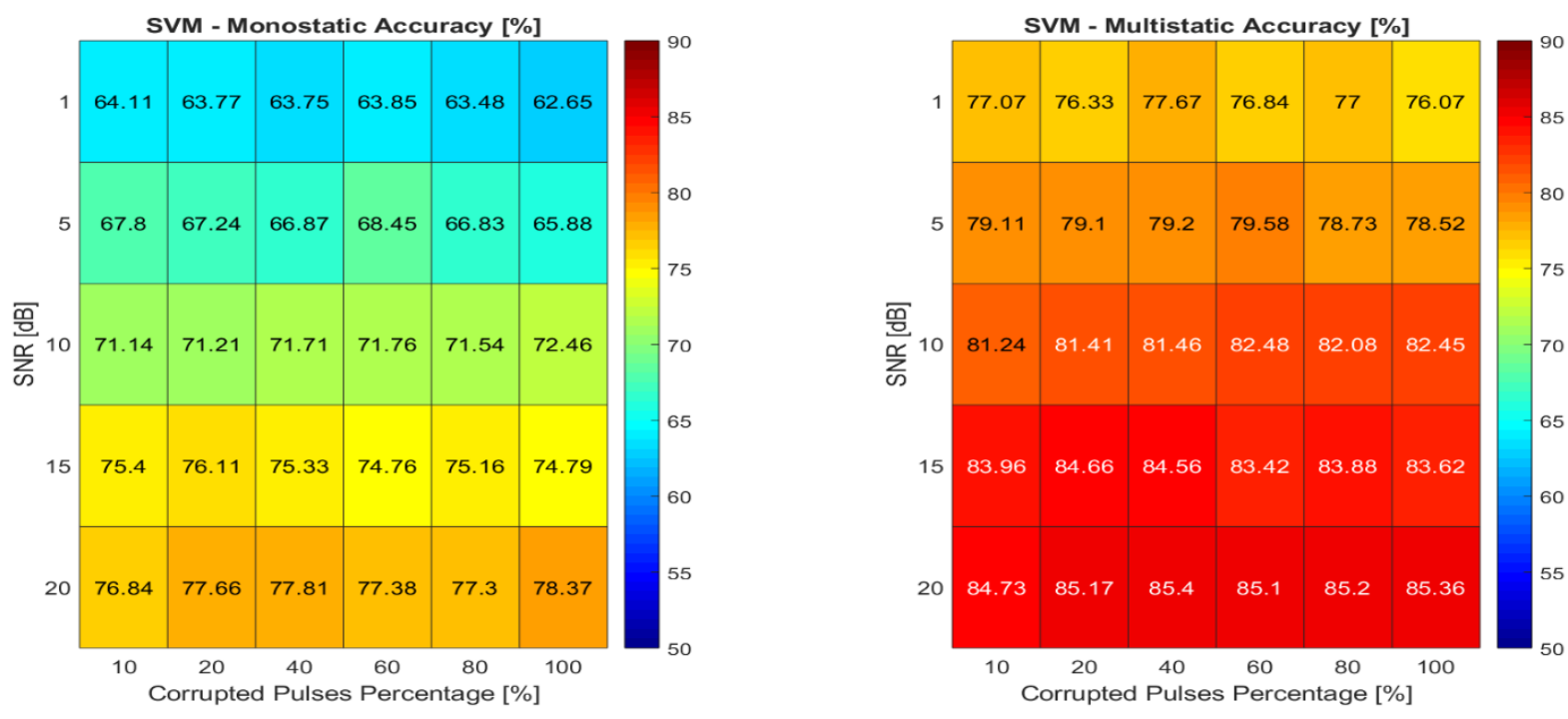

Fig. 4. Heatmaps of classification accuracy with SVM classifier comparing monostatic (left) and multistatic (right) data as a function of SNR and percentage of pulses whose SNR has been altered

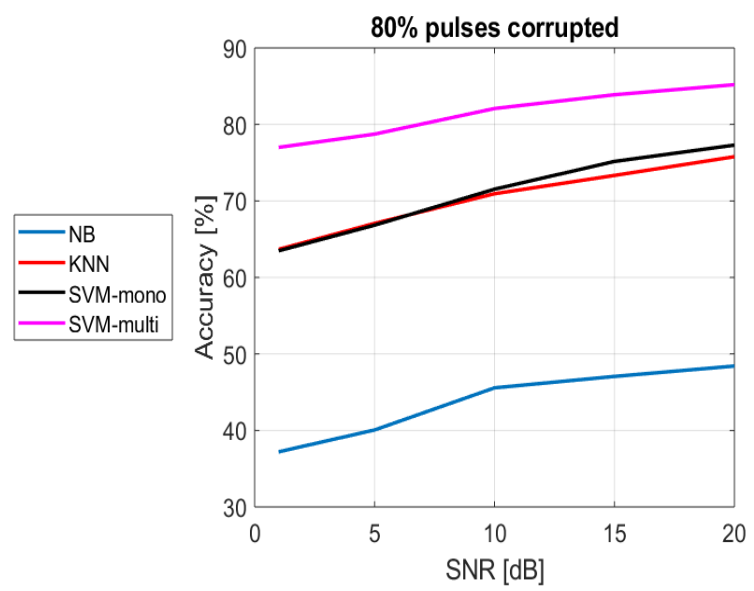

Fig. 5. Classification accuracy for different classifiers as function of SNR $-80 \%$ of the original pulses have been modified in terms of SNR

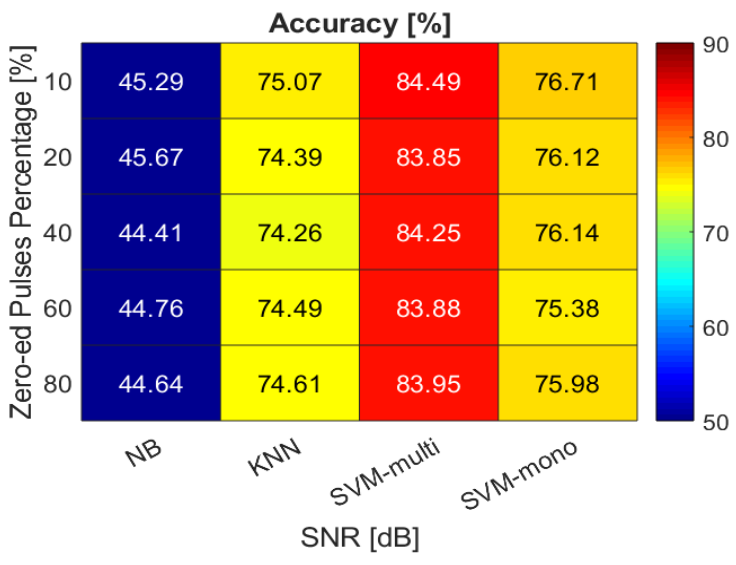

Fig. 6. Classification accuracy for different classifiers and different percentages of received radar pulses that have been forced to zero 


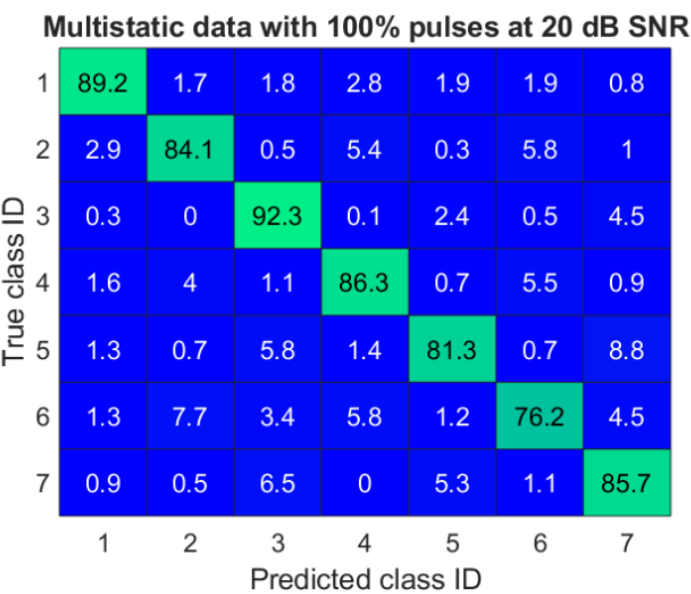

Fig. 7. Example of confusion matrix with SVM classifier and multistatic data, with $100 \%$ pulses altered at $20 \mathrm{~dB}$ SNR

\section{CONCLUSIONS}

In this paper, we evaluated the performance of multistatic human micro-Doppler for classification when the data are corrupted by some form of degradation. The majority of the works in the open literature for classification appears to assume that the quality of the radar data is good, i.e. pulses are not missing or they all have a given SNR because of the experimental and scenario conditions. In these preliminary results we started to evaluate the effect of degrading subsets of radar pulses by either changing their SNR (for example mimicking the effect of an involuntary competing transmitter near the radar), or completely replacing them with zeroes (for example mimicking malfunctioning events that make batches of pulses unusable).

The tests have been performed on experimental data collected by a multistatic S-band radar made of 3 nodes, with a 7-class problem aiming to identify seven different human walking activities, i.e. walking while carrying different objects with different postures. Although preliminary, these results show that the classification accuracy of the overall system can be reduced, especially if many radar pulses have degraded SNR. The possibility to restore and/or improve performances by using multistatic data have also been briefly explored with a simple decision-level fusion scheme.

This initial work can be expanded towards a variety of directions, considering more complicated and non-random jamming schemes, i.e. those where a long batch of consecutive pulses are degraded and more subtle degradation occurs, for example with replica or mismatched radar pulses. More robust classification schemes can be tested in order to improve the overall classification accuracy (currently at about $85 \%$ in the best case), for example those based on deep learning and data-driven feature extraction and selection, as well as more elaborated fusion schemes for multistatic information. It is envisaged that these can also provide more resilience to the system in case of the other aforementioned jamming schemes. Finally, methods to retrieve the information degraded by jamming can be explored, for example looking at transfer learning or dictionary based methods, whereby the radar could realise that some information has been damaged, and retrieve or regenerate it based on a database of previous knowledge.

\section{ACKNOWLEDGEMENT}

The authors are grateful for the support of the IET A. F. Harvey Prize 2013, awarded to Prof Hugh Griffiths. F. Fioranelli' $s$ work for processing these data was also supported by the UK EPSRC, grant EP/R041679/1.

\section{REFERENCES}

[1] M. G. Amin, Y. D. Zhang, F. Ahmad, and K. C. D. Ho, "Radar signal processing for elderly fall detection: The future for in-home monitoring," IEEE Signal Process. Mag., vol. 33, no. 2, pp. 71-80, 2016.

[2] M. S. Seyfioğlu, A. M. Özbayoğlu and S. Z. Gürbüz, "Deep convolutional autoencoder for radar-based classification of similar aided and unaided human activities," in IEEE Transactions on Aerospace and Electronic Systems, vol. 54, no. 4, pp. 1709-1723, Aug. 2018.

[3] E. Cippitelli, F. Fioranelli, E. Gambi, and S. Spinsante, "Radar and RGB-depth sensors for fall detection: a review," IEEE Sens. J., vol. 17, no. 12, pp. 3585-3604, 2017.

[4] K. Youngwook, H. Sungjae, K. Jihoon, 'Human detection using Doppler radar based on physical characteristics of targets', IEEE Geoscience and Remote Sensing Letters, vol. 12, pp. 289-293, 2015.

[5] R. Ricci, A. Balleri, 'Recognition of humans based on radar microDoppler shape spectrum features', IET Radar, Sonar \& Navigation, vol. 9 (9), pp. 1216-1223, December 2015.

[6] S. Björklund, H. Petersson and G. Hendeby, 'On distinguishing between human individuals in micro-Doppler signatures', 14th International Radar Symposium, Dresden, June 2013, pp. 865-870.

[7] F. Fioranelli, M. Ritchie, S. Z. Gürbüz, and H. Griffiths, "Feature diversity for optimized human micro-Doppler classification using multistatic radar,” IEEE Trans. Aerosp. Electron. Syst., vol. 53, no. 2, pp. 640-654, 2017.

[8] F. Fioranelli, M. Ritchie and H. Griffiths, "Centroid features for classification of armed/unarmed multiple personnel using multistatic human micro-Doppler," in IET Radar, Sonar \& Navigation, vol. 10, no. 9, pp. 1702-1710, 122016.

[9] K. Youngwook, L. Hao, 'Human activity classification based on micro-Doppler signatures using a Support Vector Machine', IEEE Transactions on Geoscience and Remote Sensing, vol. 47, pp. 13281337, 2009.

[10] S. Z. Gürbüz, B. Erol, B. Çağlıyan, B. Tekeli, 'Operational assessment and adaptive selection of micro-Doppler features', IET Radar, Sonar \& Navigation, vol. 9 (9), p. 1196-1204, December 2015.

[11] D. P. Fairchild, R. M. Narayanan, 'Multistatic micro-Doppler radar for determining target orientation and activity classification', IEEE Transactions on Aerospace and Electronic Systems, vol. 52, no. 1, pp. 512-521, February 2016

[12] W. L. van Rossum, L. Anitori, P. van Dorp, J. J. M. de Wit and R. I. A. Harmanny, "Classification of human gaits using interrupted radar measurements," 2017 IEEE Radar Conference (RadarConf), Seattle, WA, 2017, pp. 0514-0519.

[13] G.W. Stimson, H. Griffiths, C. Baker, D. Adamy, 'Stimson's Introduction to Airborne Radar', 3rd Edition, The Institution of Engineering and Technology, 2014

[14] T. E. Derham, S. Doughty, K. Woodbridge, C. J. Baker, 'Design and evaluation of a low-cost multistatic netted radar system', IET Radar, Sonar \& Navigation, vol. 1, pp. 362-368, 2007.

[15] B. Erol, M. G. Amin and S. Z. Gurbuz, "Automatic Data-Driven Frequency-Warped Cepstral Feature Design for Micro-Doppler Classification," in IEEE Transactions on Aerospace and Electronic Systems, vol. 54, no. 4, pp. 1724-1738, Aug. 2018. 